\title{
Brocca et al.
}

Protein adsorption at the air-water interface by a charge sensing interferometric technique.

\section{SUPPORTING INFORMATION}

\section{A) TRANSITION EVENT BY ACOUSTIC EXCITATION}

Bubble acoustic excitation was obtained by applying a piezo-electric transducer installed at the bottom of the measuring cell.

Figure S1 shows the behaviour of the resonance frequency of a SDS covered bubble as a function of SDS bulk concentration. The first experimental points from 0 to $0.03 \mathrm{mM}$ concentration show a stable frequency in agreement with the negligible effect on surface properties seen by other methods at those concentrations. A transition event follows, in perfect analogy with what seen by e.m. excitation (Figure 6 in the main text) eventually resulting in a downward shift at about $0.045 \mathrm{mM}$ concentration.

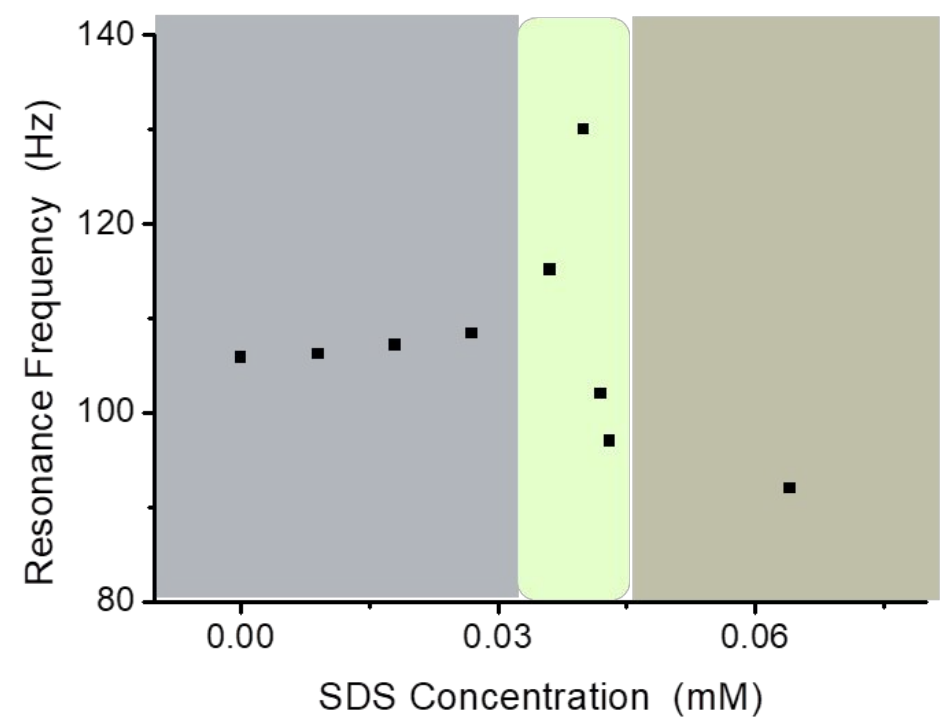

Figure $\mathrm{S1}$. Frequency of the $\mathrm{I}=1$ mode of a $\mathrm{R}=0.78 \mathrm{~mm}$ bubble excited by acoustic waves as a function of the bulk concentration of SDS.

B) IRROTATIONAL MODEL FOR A SURFACTANT COVERED BUBBLE (DROP) UNDERGOING SHAPE OSCILLATIONS. 
Following Padrino, Funada and Joseph (Ref. 57 of the main text), two immiscible fluids are arranged in a way to form a spherical drop at rest. Later on, by tending to zero the density of the inner fluid, we may also describe the behavior of a bubble. For a pure irrotational flow, the incompressible Navier-Stokes equations reduce to the simpler Bernoulli's equation. Ignoring non-linear terms, the resulting pressure field inside the drop (or bubble) can be decomposed into the undisturbed pressure $P_{\text {in }}$ plus a small disturbance $p_{\text {in }}$.

$$
p_{i n}=-\rho_{\text {in }} \frac{\partial \phi_{i n}}{\partial t}
$$

$\nabla^{2} \phi_{i n}=0$

where $\phi_{i n}$ is the so-called velocity potential, $R_{o}$ the radius of the drop (bubble) at rest, $\rho_{i n}$ is the inner fluid density and $\nabla^{2}$ is the spherical Laplacian operator:

$$
\nabla^{2} \equiv \frac{\partial^{2}}{\partial r^{2}}+\frac{2}{r} \frac{\partial}{\partial r}+\frac{1}{r^{2}} \nabla_{\perp}^{2}=\frac{\partial^{2}}{\partial r^{2}}+\frac{2}{r} \frac{\partial}{\partial r}+\frac{1}{r^{2}}\left(\frac{1}{\sin \theta} \frac{\partial}{\partial \theta}\left(\sin \theta \frac{\partial}{\partial \theta}\right)+\frac{1}{\sin ^{2} \theta} \frac{\partial^{2}}{\partial \varphi^{2}}\right)
$$

Analogously, in the outer region $R_{o}<r<\infty$ we have:

$$
\begin{aligned}
& p_{\text {out }}=-\rho_{\text {out }} \frac{\partial \phi_{\text {out }}}{\partial t} \\
& \nabla^{2} \phi_{\text {out }}=0
\end{aligned}
$$

$$
R_{o}<r<\infty
$$

We seek at a general solution to eqs.( $(\mathrm{S} 1 \mathrm{~b})$ and $(\mathrm{S} 3 \mathrm{~b})$ satisfying the boundary conditions: $\lim _{r \rightarrow \infty} \phi_{\text {out }}=0$ and $\lim _{r \rightarrow 0} \phi_{\text {in }}=$ finite:

$$
\begin{array}{rlrl}
\phi_{\text {in }} & =\sum_{l=0}^{\infty} A_{l}\left(\frac{r}{R_{o}}\right)^{l} e^{-\Omega_{l} t} S_{l}(\theta, \varphi)+C . C . & 0<r<R_{o} \\
\phi_{\text {out }}=\sum_{l=0}^{\infty} C_{l}\left(\frac{r}{R_{o}}\right)^{-l-1} e^{-\Omega_{l} t} S_{l}(\theta, \varphi)+C . C . & R_{o}<r<\infty
\end{array}
$$


where $A_{l}, C_{l}$ and $\Omega_{l}$ are still unknown parameters to be determined by applying the proper boundary conditions and C.C. designates the conjugate complex. $S_{l}(\theta, \varphi)$ are surface harmonics of integer order:

$$
S_{l}(\theta, \varphi)=\sum_{m=-l}^{l} B_{l m} Y_{l}^{m}(\theta, \varphi)
$$

The functions $Y_{l}^{m}(\theta, \varphi)$ are defined as: $Y_{l}^{m}(\theta, \varphi)=P_{l}^{|m|}(\cos \theta) e^{i m \varphi}$, where $P_{l}^{m}(\cos \theta)$ are associate Legendre functions. $Y_{l}^{m}(\theta, \varphi)$ satisfy the equation:

$\nabla_{\perp}^{2} Y_{l}^{m}(\theta, \varphi)=-. l(l+1) Y_{l}^{m}(\theta, \varphi)$

for $I=0,1,2 \ldots \ldots$ and $|m| \leq l$. The above relationship that will be repeatedly used in the following calculations.

Solutions (S4) must satisfy the proper boundary conditions. The first one requires continuity of the radial velocity at the interface. Moreover, at the interface, the velocities of both fluids must equate that $(\partial \varsigma / \partial t)$ of the moving boundary:

$\left.u_{r}^{\text {in }}\right|_{r=R_{o}}=\left.u_{r}^{\text {out }}\right|_{r=R_{o}}=\frac{\partial \zeta}{\partial t}$

Notice that for irrotational motions, the components of the velocity vector are simply related to the potential gradient, so: $u_{r}=\partial \phi / \partial r$.

It is convenient to expand also the local surface deformation $\varsigma$ from the equilibrium value $R_{o}$ in a series of surface harmonics: $R=R_{o}+\varsigma$, where:

$\varsigma=\sum_{l=0}^{\infty} \varsigma_{o l} S_{l}(\theta, \varphi) e^{-\Omega_{l} t}+$ C.C.

Inserting eqs.(S4) into eqs.(S7), we may express the coefficients $A_{l}$ and $C_{l}$ as a function of $\Omega_{l}$ :

$\frac{l}{R_{o}} A_{l}=-\Omega_{l} \zeta_{o l} \quad \frac{l+1}{R_{o}} C_{l}=\Omega_{l} \zeta_{o l}$

The second boundary condition arises from the balance of normal stresses across the interface. Using the explicit formula for the normal stress, we find:

$\left[-P_{\text {in }}-p_{\text {in }}+2 \mu_{\text {in }} \frac{\partial u_{r}^{\text {in }}}{\partial r}+P_{\text {out }}+p_{\text {out }}-\mu_{\text {out }} \frac{\partial u_{r}^{\text {out }}}{\partial r}\right]_{r=R_{o}}=\sigma H$

where $\mu_{\text {in }}$ and $\mu_{\text {out }}$ are the dynamic viscosities in the inner and outer fluids, respectively. $H$ is a measure of the local curvature of the deformed drop (bubble): 


$$
H=-\frac{2}{R_{o}}+\frac{1}{R_{o}^{2}}\left(2+\nabla_{\perp}^{2}\right) \varsigma+O\left(R_{o}^{-3}\right)
$$

where $\nabla_{\perp}^{2}$ has been defined in eq (S2). Combining now eqs (S10) and (S11), eliminating $p_{\text {in }}$ and $p_{\text {out }}$ by eqs ( $\mathrm{S} 1 \mathrm{a}$ ) and (S3a) and using eq (S6) to calculate the term $\left(2+\nabla_{\perp}^{2}\right) \varsigma$ in eq.(S11), we get after a little algebra:

$$
\begin{aligned}
& P_{\text {in }}-P_{\text {out }}=\frac{2 \sigma}{R_{o}} \\
& \left(\rho_{\text {in }}(l+1)+\rho_{\text {out }} l\right) \Omega_{l}^{2}-\frac{2}{R_{o}^{2}}\left(\mu_{\text {in }}(l+1) l(l-1)+\mu_{\text {out }}(l+2)(l+1) l\right) \Omega_{l}+\frac{\sigma}{R_{o}^{3}}(l+2)(l+1) l(l-1)=0
\end{aligned}
$$

Equation (S12a) in nothing but the classical Young-Laplace relationship for a spherical bubble. Equation (S12b) instead represents the wanted dispersion relationship for the eigenvalues $\Omega_{l}$.

In the case of bubbles, both the gas density and its viscosity are negligible, so in eq (S12b) we may put: $\rho_{i n} \approx \mu_{i n} \approx 0$.

Solving eq (S12b) with respect to $\Omega_{l}$ yields complex numbers. The real part of $\Omega_{l}$ , $\operatorname{Re} \Omega_{l}$, accounts for the decay of a generic shape deformation (imposed at $t=0$ ) toward the equilibrium spherical shape. A slow relaxation rate leads to narrow resonance peaks in our "bubble spectroscopy", while a fast relaxation rate (i.e., strong viscous damping) produces a considerable broadening and, eventually, the disappearance of the resonance peak. On the contrary, the imaginary part of $\Omega_{l}, \omega_{o l}=\operatorname{Im} \Omega_{l}$, fixes the numerical value of the oscillation eigen-frequencies. Albeit the solution of eq (S12b) is trivial, a more transparent result is achieved noticing that the linear term in $\Omega_{l}$ is small for any reasonable values of the different physical parameters, so, from eq (S12b) eventually we find in the case of a gas bubble:

$\omega_{\text {ol }}=\operatorname{Im} \Omega_{l}=\left[\frac{\sigma}{\rho_{\text {out }} R_{o}^{3}}(l+2)(l+1)(l-1)\right]^{1 / 2} \quad$ for $\quad l \geq 2$

which is coincident with the relationship (1b) reported in the main text provided the anchoring effects accounted for by the $D(l, a)$ term have been neglected.

Notice that eq (S13) applies for $l \geq 2$, while for $l=1$ we get: $\omega_{o l}=0$. This result is not surprising since the motion with $l=1$ describes a translational displacement of the whole bubble that, obviously, occurs at zero frequency. This result is 
rigorously true for a free bubble, while in our study we employ bubbles anchored to a rigid electrode. In this configuration, the motion with $l=1$ occurs at finite frequency. Unfortunately, the mathematical modeling is much more difficult and we refer to the specialized literature for further details (Refs. 19-21 of the main text).

The last step is relate the surface tension $\sigma$ to the adsorption of surface active molecules. In the case of uncharged species, this goal can be reached by the usual procedures sketched in many articles and textbooks. Here we develop a simple variational approach particularly suitable to include the coupling between the electrostatic effects and the local variations of the curvature radius of the interface as discussed in SM C.

The total free energy of the system, $F \equiv F\left[C^{+}\right]$, is described by a local functional $\Delta f\left(C^{+}\right)$that depends on the protein concentration profile $C^{+}(r, \theta, \phi)=C^{+}$ (dimensionless units) and accounts for the non-electrostatic energy components of the protein in the bulk (we assumed the protein to be positive, results do not change if we consider a negatively charged protein). This contribution must be implemented by another term, $f^{\text {suf }}\left(\left.C^{+}\right|_{R}\right)$, that takes into account the nonelectrostatic energy of the adsorbed specie at the interface. We also assume that only proteins are surface-active, while the negative counter-ions, as well as added salts or $\mathrm{H}^{+}$and $\mathrm{OH}^{-}$coming from water self-dissociation, do not adsorb at the interface. Setting the origin at the center of the bubble and letting $R=R(\theta, \phi)$ be the local radius of the deformed sphere, the free energy functional $F \equiv F\left[C^{+}\right]$ takes the form:

$F\left[C^{+}\right]=4 \pi \int_{R}^{\infty}\left[\Delta f\left(C^{+}\right)\right\}^{2} d r+f^{\text {surf }}\left(\left.C^{+}\right|_{R}\right) S$

where $C^{+}(r, \theta, \phi) \equiv C^{+}$is the protein concentration. In spherical polar coordinates, the surface area $S$ of the deformed sphere is given by: $\int_{0}^{2 \pi} \int_{0}^{\pi} \sqrt{g} R^{2} \sin \theta d \theta d \phi=S$, where the metric $\sqrt{g}$ reads: $\sqrt{g}=\left(1+\frac{1}{R^{2}}\left(\frac{\partial R}{\partial \theta}\right)^{2}+\frac{1}{R^{2} \sin ^{2} \theta}\left(\frac{\partial R}{\partial \phi}\right)^{2}\right)^{1 / 2}$. Equation (S14) represents a variational formulation of the interfacial energy calculation. Similar approaches had been developed by many authors, here we extended the procedure to deformed spherical surfaces. As we will show soon, results are identical to those obtained for a flat surface, they, however, will be different when long-range electrostatic interactions are considered. The functional $\Delta f\left(C^{+}\right)$describes the non- 
electric part of the free energy variation on going from a distance $r$ to the infinity, its analytical expression reads:

$$
a^{3} \Delta f\left(C^{+}\right) \approx k_{B} T\left[C^{+}\left(\log C^{+}-1\right)-\bar{C}(\log \bar{C}-1)\right]-\mu_{b}^{+}\left(C^{+}-\bar{C}\right)
$$

$\mu_{b}^{+}$being the still unknown chemical potential of the protein in the bulk and $a^{3}$ the unit volume. The overbar notation $\bar{C}$ labels the concentration of the surface-active molecule at large distance. The functional $f^{\text {suf }}\left(\left.C^{+}\right|_{R}\right)$ describes the energy contribution at the interface $r \rightarrow R(\theta, \phi)$ :

$$
\begin{aligned}
a^{2} f^{\text {suf }}\left(\left.C^{+}\right|_{R}\right) \approx & k_{B} T\left[\left.\left.C^{+}\right|_{R} \log C^{+}\right|_{R}+\left(1-\left.C^{+}\right|_{R}\right) \log \left(1-\left.C^{+}\right|_{R}\right)\right]- \\
& -\left.\left(\lambda+\left.\mu^{+}\right|_{R}\right) C^{+}\right|_{R}-\beta\left(\left.C^{+}\right|_{R}\right)^{2} \quad(\mathrm{~S} 15 \mathrm{~b})
\end{aligned}
$$

Since the protein concentration at the bubble surface, $\left.C^{+}\right|_{R}$, can be large, in writing the entropic contribution at the interface, eq (S15b), we retained the full expression, while the approximate expression, valid in the dilute solution limit, was employed in the dilute bulk phase (eq (S15a)). The parameter $\lambda$ accounts for the hydrophobic forces that push the protein at the air-water interface, while $\beta$ plays the role of a cooperativity parameter proportional to the strength of protein-protein interactions. Lastly, $\left.\mu^{+}\right|_{R}$ is the protein chemical potential calculated at the interface $r \rightarrow R(\theta)$. Minimization of the functional (S14) with respect to $C^{+}$, enables us, with the aid of eq.(S15a), to calculate the chemical potential: $\mu_{b}^{+}(r \rightarrow \infty)=k_{B} T \log \bar{C}$. At equilibrium, we must have:

$$
\left.\mu^{+}\right|_{R}=\mu_{b}^{+}(r \rightarrow \infty)
$$

Hence, letting $\frac{\left.C^{+}\right|_{R}}{a^{2}} \equiv \Gamma$ be the 2D protein surface concentration $\left(m o l e \cdot m^{-2}\right)$, and $\left.\Gamma_{\max } \approx \lim _{C^{+} \rightarrow 1} C^{+}\right|_{R} / a^{2}$ be the maximum allowed 2D concentration at full coverage, we find that $\Gamma$ is related to the bulk protein concentration $\bar{C}$ by the relationship:

$$
\frac{\Gamma}{\Gamma_{\max }}=\frac{\bar{C}}{\bar{C}+K \exp \left(-\frac{2 \beta}{k_{B} T} \frac{\Gamma}{\Gamma_{\max }}\right)}
$$


where $K \equiv e^{-\lambda / k_{B} T}$ is the binding constant of the surface-active molecule to the airwater interface. This formula coincides with the celebrated Frumkin's isotherm. As we can see, in absence of electrostatic forces, surface adsorption does not depend upon the counter-ion concentration (provided they do not adsorb at the interface). This conclusion turns out to be wrong when one introduces long-range electrostatic forces as shown in the next section. Inserting the explicit expression for $\Gamma$ (eq (S17)) into the energy functional (S14), we find an expression for the surface tension $\sigma-\sigma_{o}$ , where $\sigma_{o}$ is the surface tension of the solvent in the absence of surface-active molecules. For uncharged systems, the local surface tension can be written as: $a^{2}\left(\sigma-\sigma_{o}\right)=\frac{1}{N_{S}} F\left[C^{+}\right]_{\text {optimized }}$, where $N_{S}=S / a^{2} \approx 4 \pi R_{o}^{2} / a^{2}$ is the number of sites at the interface of a deformed sphere. That is:

$\sigma-\sigma_{o}=\Gamma_{\max }\left[k_{B} T \log \left(1-\Gamma / \Gamma_{\max }\right)+\beta\left(\Gamma / \Gamma_{\max }\right)^{2}\right]$

\section{C) "APPARENT" SURFACE TENSION OF BUBBLES UNDERGOING SHAPE OSCILLATIONS.}

In order to describe electrostatic effects on oscillating interfaces, we have to introduce a dependence of the local surface energy on the local curvature radius. The physical rationale is grounded on the long-range nature of the electrostatic forces that leads to a relationship between the electrostatic and the local curvature radius of the interface.

The starting point is a general expression for the surface free energy functional described, in the present case, as depending on several degrees of freedom: the dimensionless protein concentration profile, $C^{+}(r, \theta, \phi)$ (we arbitrarily assume that the protein bears a $\mathrm{pH}$-dependent excess of $Z_{+}$positive charges, results do not change by assuming a negative charge excess). Moreover, $C_{i}^{ \pm}(r, \theta, \phi)$ is the profile of any charged specie that does not adsorb at the interface (i.e., negative counter-ions, eventual added salts, $\mathrm{H}^{+}$and $\mathrm{OH}^{-}$ions coming from water self-dissociation). Finally, $\Phi(r, \theta, \phi)$ is the electrostatic potential generated by the surface charges. Setting the 
origin at the center of the bubble, letting $R=R(\theta, \phi)$ be the local radius of the deformed sphere and assuming that only the positive specie (protein) is surfaceactive, the total free energy functional takes the form:

$$
\begin{gathered}
F\left[C^{+}, C_{i}^{ \pm}, \Phi\right]=4 \pi \int_{R}^{\infty}\left[\Delta f\left(C^{+}\right)+\sum_{i} \Delta f_{i}\left(C_{i}^{ \pm}\right)+f_{e l}\left(C^{+}, C_{i}^{ \pm}, \Phi\right)\right] r^{2} d r+ \\
\left|f^{\text {suff }}\left(\left.C^{+}\right|_{R}\right)+f_{e l}^{\text {suff }}\left(\left.C^{+}\right|_{R}\right)\right| S
\end{gathered}
$$

where, for the sake of compactness, the $r, \theta$ and $\phi$-dependence of $C^{+}, C_{i}^{ \pm}$, and $\Phi$ has been omitted, while the deformed bubble surface area $S$ has been defined just after eq.(S14). The functionals $\Delta f\left(C^{+}\right), \Delta f_{i}\left(C_{i}^{ \pm}\right)$and $f^{\text {surf }}\left(\left.C^{+}\right|_{R}\right)$ refer to an uncharged system and have been previously defined by eqs (S15a,b) in SM B. The new energy functionals $f_{e l}\left(C^{+}, C_{i}^{ \pm}, \Phi\right)$ and $f_{e l}^{s u f}\left(\left.C^{+}\right|_{R}\right)$ account for the electrostatic effects, both in the bulk and surfaces phases, their explicit expressions are:

$$
\begin{aligned}
f_{e l}\left(C^{+}, C_{i}^{ \pm}, \Phi\right) & =\frac{e}{a^{3}}\left[Z_{+} C^{+} \pm \sum_{i} C_{i}^{ \pm}\right] \Phi-\frac{\varepsilon}{8 \pi}\left(\frac{\partial \Phi}{\partial n}\right)^{2} \\
f_{e l}^{\text {sup }}\left(\left.C^{+}\right|_{R}\right) & =\frac{e}{a^{2}}\left[\left.\left(Z_{+} C^{+}\right] \Phi\right|_{R}\right.
\end{aligned}
$$

where $\varepsilon$ is the dielectric permittivity of water and $\left(\frac{\partial \Phi}{\partial n}\right)$ is the derivative of the electrostatic potential with respect to the normal to the interface. Minimization of the functional (S19) was performed by the standard Euler-Lagrange equations. Variation with respect to $C^{+}$and $C^{-}$, together with the use of the electroneutrality condition for protein and its monovalent counter-ions (i.e., $C^{-}(r \rightarrow \infty)=Z_{+} C^{+}(r \rightarrow \infty)$ $\equiv Z_{+} \bar{C}$ ), yields the expected Boltzmann formula for protein and counter-ions profiles:

$C^{+}=\bar{C} \exp \left(-\frac{Z_{+} e \Phi}{k_{B} T}\right), \quad C^{-}=Z_{+} \bar{C} \exp \left(+\frac{e \Phi}{k_{B} T}\right)$

Similarly, the profile of any other $i$-th univalent ion reads:

$$
C_{i}^{ \pm}=\bar{C}_{i}^{ \pm} \exp \left(\square \frac{e \Phi}{k_{B} T}\right)
$$


Following the same procedure, variation of the energy functional (S19) with respect to $\left.\mathrm{C}^{+}\right|_{R}$ and introducing the 2D protein surface concentration $\left(\mathrm{mole} \cdot \mathrm{m}^{-2}\right) \frac{\left.\mathrm{C}^{+}\right|_{R}}{a^{2}} \equiv \Gamma$, we find the relationship:

$$
\frac{\Gamma}{\Gamma_{\max }}=\frac{\bar{C}}{\bar{C}+K \exp \left(-\frac{2 \beta}{k_{B} T} \frac{\Gamma}{\Gamma_{\max }}+\left.\frac{Z_{+} e}{k_{B} T} \Phi\right|_{R}\right)}
$$

Which is nothing but the Davies isotherm. In the limit $\left.\Phi\right|_{R} \rightarrow 0$, we recover the Frumkin isotherm, eq (S17). Notice that the electrostatic potential calculated at the interface, $\Phi_{R}$, is itself a function of $\Gamma$ as it will be shown shortly.

The most difficult part is the calculation of the electrostatic potential $\Phi=\Phi(r, \theta, \phi)$. By employing the Euler-Lagrange equation, variation with respect to $\Phi$ and its spacederivative leads to the Poisson-Boltzmann equation:

$$
\nabla^{2} \Phi=-\frac{4 \pi e}{\varepsilon a^{3}}\left[Z_{+} \bar{C}\left[\exp \left(-\frac{Z_{+} e \Phi}{k_{B} T}\right)-\exp \left(+\frac{e \Phi}{k_{B} T}\right)\right] \pm \sum_{i} \bar{C}_{i}^{ \pm} \exp \left(\square \frac{e \Phi}{k_{B} T}\right)\right]
$$

where the operator $\nabla^{2}$ in spherical polar coordinates has been defined by eq (S2). Equation (S23) admits simple solutions only in the Debye-Huckel limit (small $\Phi$ ). In this case, a series expansion of the exponential functions leads to a solvable linear differential equation:

$$
\nabla^{2} \Phi=\kappa^{2} \Phi \quad(\mathrm{S} 24)
$$

with $\kappa^{2} \equiv 4 \pi e^{2} \frac{\left(Z_{+}^{2}+\left|Z_{+}\right|\right) \bar{C}+\sum_{i} \bar{C}_{i}^{ \pm}}{\varepsilon k_{B} T a^{3}}$ for a $Z_{+}: 1$ protein-counterions system in the presence of other 1:1 electrolytes.

Lastly, variation of the functional (S19) with respect to $\left.\Phi\right|_{R}$ provides a boundary condition to the linearized Poisson-Boltzmann equation (S24). Following the standard procedures of the variational calculus we obtain:

$\left.\frac{\varepsilon}{4 \pi} \frac{\partial \Phi}{\partial n}\right|_{R}=-e Z_{+} \frac{\left.C^{+}\right|_{R}}{a^{2}} \equiv-e Z_{+} \Gamma$ 
where we have introduced the $2 \mathrm{D}$ interfacial concentration $\left.\Gamma \equiv C^{+}\right|_{R} / a^{2}$. Equation (S24) is solvable, simple analytical solutions arise only for regular geometries. Our goal is to extend the classical results to a slightly deformed sphere. This problem has been addressed by several authors (see, e.g., Jyh Ping Hsu, Bo Tau Liu, Exact solution to the linearized Poisson-Boltzmann equation for spheroidal surfaces J. Colloid Interface Sci. 1996, 178, 785-788; Alvarez, C., Tellez, G. Screening of charged spheroidal colloidal particles J. Chem. Phys. 2010, 133, 144908). Results, however, are often unnecessarily complicated and particularly suitable for large deformations. In the limit of small deformations, some papers produce simpler approximate results. Here we generalize a procedure set forth by Ohshima for a spheroid (Ohshima, H. Double-layer potential distribution and surface charge density/surface potential relationship for a nearly spherical spheroid in an electrolyte solution, Colloids Surf. A, 2000, 169, 13-16).

For small deviations from the spherical shape, the electrostatic potential $\Phi$ does not depend appreciably on $\theta$ and $\phi$, resulting similar to that calculated for a perfect sphere: $\nabla^{2} \Phi \approx \frac{\partial^{2} \Phi}{\partial r^{2}}+\frac{2}{r} \frac{\partial \Phi}{\partial r}$ (see eq (S2)). So, eq (S24) reduces to:

$\frac{\partial^{2} \Phi}{\partial r^{2}}+\frac{2}{r} \frac{\partial \Phi}{\partial r}=\kappa^{2} \Phi$

Equation (S26) admits exact analytical solution satisfying the boundary condition $\underset{r \rightarrow \infty}{\rightarrow} 0$ :

$\Phi=\Phi_{o} \frac{R_{o}}{r} e^{-\kappa\left(r-R_{o}\right)}$

The still unknown constant $\Phi_{o}$, the interfacial surface potential $\left(\operatorname{ch} \arg e \cdot m^{-1}\right)$, can be calculated by imposing the boundary condition (S25). Approximating $\left.\left.\frac{\partial \Phi}{\partial n}\right|_{R} \approx \frac{\partial \Phi}{\partial r}\right|_{R_{o}}$ (they differ by quadratic terms in $\zeta$ ), we find:

$\Phi_{o}=\frac{4 \pi Z_{+} e \Gamma}{\varepsilon \kappa\left(1+\frac{1}{\kappa R_{o}}\right)}$ 
Equations (S27) and (S28) apply to a regular charged sphere of radius $R_{o}$, our goal is to extend this result to a slightly deformed sphere. In the most general case where $\Phi=\Phi(r, \theta, \phi)$, solution to the linearized Poisson-Boltzmann equation (S24) satisfying the condition $\Phi \underset{r \rightarrow \infty}{\rightarrow} 0$ reads:

$$
\Phi=H_{o} \frac{R_{o}}{r} e^{-\kappa\left(r-R_{o}\right)}+\sum_{l=2}^{\infty} H_{l} \aleph_{l}(\kappa r) S_{l}(\theta, \phi)
$$

where the dimensionless $r$-dependent terms, $\aleph_{l}(k r)$, are modified spherical Bessel functions of the second kind (viz.: $\left.\aleph_{l}(\kappa r) \equiv \sqrt{\frac{R_{o}}{r}} K_{l+1 / 2}\left(\kappa\left(r-R_{o}\right)\right)\right)$, while $H_{o}$ and $H_{l}($ charg $\left.e \cdot \mathrm{m}^{-1}\right)$ are coefficients to be determined and $S_{l}(\theta, \phi)$ are surface harmonics defined by eq (S5). By exploiting the boundary condition (S25), we get:

$$
\left[-H_{o} \frac{R_{o}}{r^{2}}(1+\kappa r) e^{-\kappa\left(r-R_{o}\right)}+\sum_{l=2}^{\infty} H_{l} \frac{\partial \aleph_{j}(k r)}{\partial r} S_{l}(\theta, \phi)\right]_{r \rightarrow R}=-\frac{4 \pi}{\varepsilon} e Z_{+} \Gamma
$$

The summation in the left hand side of eq.(S30) must vanish for a regular sphere, so $H_{l}$ must be small and proportional to the maximum deformation $\zeta_{o l}$. When $r$ approaches to the deformed spherical surface $R(\theta, \phi)=R_{o}+\zeta$, we can calculate from eq.(S30) $H_{o}$ and $H_{l}$. Up to linear terms in $\zeta$ we find:

$$
H_{o}=\frac{4 \pi Z_{+} e \Gamma}{\varepsilon \kappa\left(1+\frac{1}{\kappa R_{o}}\right)}, \quad H_{l}=-\frac{8 \pi Z_{+} e \Gamma}{\varepsilon} \cdot \frac{1+\kappa R_{o}+\frac{1}{2} \kappa^{2} R_{o}^{2}}{R_{o}\left(1+\kappa R_{o}\right)}\left(\left.\frac{\partial \aleph_{l}(\kappa r)}{\partial r}\right|_{R_{o}}\right)^{-1} \zeta_{o l} e^{-\Omega, t}+C . C .
$$

where, as expected, $H_{o}=\Phi_{o}$ (see eq (S28)). Replacing eq (S31) into eq (S29) and expanding again in power series of $\zeta$, we obtain the searched expression for the surface potential of a slightly deformed sphere:

$$
\left.\Phi\right|_{R}=\Phi_{o}-\frac{\Phi_{o}}{R_{o}} \sum_{l=2}^{\infty}\left[1+\kappa R_{o}+\left.\frac{2}{R_{o}^{2}}\left(1+\kappa R_{o}+\frac{1}{2} \kappa^{2} R_{o}^{2}\right) \frac{\aleph_{l}(\kappa r)}{\partial \aleph_{l}(\kappa r) / \partial r}\right|_{R_{o}}\right] \zeta_{o l} e^{-\Omega_{l} t} S_{l}(\theta, \phi)+C . C .
$$

$\Phi_{o}$ being the electrostatic potential at the surface of a regular sphere given by eq. (S28). In calculating eq (S32) we use the asymptotic developments of the modified 
Bessel's functions valid in the limit $\kappa R_{o} \gg 1$ (Abramowitz, M., Stegun, I.A. Handbook of Mathematical Functions, Dover, New York, 1972). After some algebra we find:

$$
\frac{\aleph_{l}\left(\kappa R_{o}\right)}{\partial \aleph_{l}\left(\kappa R_{o}\right) / \partial R_{o}} \approx-\frac{2 R_{o}}{1+2 \kappa R_{o} \frac{\frac{n(n+1)+1}{2 \kappa R_{o}}+\ldots}{1+\frac{n(n+1)}{2 \kappa R_{o}}+\ldots}} \rightarrow-\frac{R_{o}}{1+\kappa R_{o}}
$$

Whence, in the limit $\kappa R_{o}>1$, eq.(32A) reduces to:

$\Phi_{R} \approx \Phi_{o}\left(1+\frac{1}{\kappa R_{o}^{2}} \sum_{l=2}^{\infty} \zeta_{o l} e^{-\Omega, t} S_{l}(\theta, \phi)+C . C.\right) \approx \Phi_{o}\left(1+\frac{\zeta}{\kappa R_{o}^{2}}\right)$

a value strongly dependent on the bubble deformation $\zeta_{o l}$ and radius $R_{o}$ but that does not appreciably varies with the oscillation mode $I$.

In deriving eq (S34) we have assumed that the surface charge density (proportional to the protein surface excess $\Gamma$ ) of an oscillating sphere remains constant during the time-dependent shape deformation. Such an assumption is correct in the limit of extremely fast shape deformations because molecules adsorbed the moving surface cannot relax toward a more favorable surface charge distribution. Different results arise adopting just the opposite picture where we maintain constant the surface potential and allows for surface charge redistribution over the oscillating surface. This picture is valid in the limit of slow motions of the interface. In the real world, the situation is in between these opposite limits. In our experiments, bubbles oscillate in the range of $10^{2} \mathrm{~Hz}$, the region of the bubble resonance. These fast deformations enable only a partial polarization of the surface charges that periodically move toward regions of greater curvature.

By repeating the same procedure adopted to derive eq (S34) we obtain a compact expression for the surface charge density $\left.\delta\right|_{R}=e Z_{+} \Gamma$ :

$\left.\delta\right|_{R} \approx \delta_{o}\left(1-\frac{1}{\kappa R_{o}^{2}} \sum_{l=2}^{\infty} \zeta_{o l} e^{-\Omega_{l} t} S_{l}(\theta, \phi)+C . C.\right)$

where $\delta_{o}$ is the surface charged density over an un-deformed sphere. As expected, the behavior is qualitatively similar to that found for the surface potential, eq.(34A). 
Let us now investigate the role of the electrostatic terms of eigen-frequencies of a bubble undergoing shape oscillations.

The first task is the calculation of the local interfacial tension defined as: $a^{2}\left(\sigma-\sigma_{o}\right)=\frac{1}{N_{S}} F\left[C^{+}\right]_{\text {optimized }}$ (see eq (S18)). Inserting the explicit expressions for $C^{+}, C^{-}$ (protein and its counter ions) and $C_{i}^{ \pm}$(other 1:1 electrolytes) into the free energy functional (S19) valid for charged systems we find:

$\sigma=\sigma_{o}+\frac{1}{a^{2}}\left[k_{B} T \log \left(1-\Gamma / \Gamma_{\max }\right)+\beta\left(\Gamma / \Gamma_{\max }\right)^{2}-\frac{a^{2}}{S} \int_{R}^{\infty}\left(A \Phi^{2}+\frac{\varepsilon}{8 \pi}\left(\frac{\partial \Phi}{\partial r}\right)^{2}\right) r^{2} d r\right]$

with $A \equiv \frac{e^{2}\left[\left(Z_{+}^{2}+\frac{1}{2}\left|Z_{+}\right|-1\right) \bar{C}+\bar{C}_{e x t}\right]}{a^{3} k_{B} T}$. The concentration $\bar{C}_{e x t}$ accounts for the double-layer effects arising from other surface-inactive ions $^{+}$and $\mathrm{OH}^{-}$in the present study, with $\bar{C}_{e x t}>\bar{C}$ ). By using the explicit expressions for the potential $\Phi$ and asymptotically integrating over $r$, we obtain in the Debye-Huckel limit:

$$
\frac{a^{2}}{S} \int_{R}^{\infty} \Phi^{2} r^{2} d r \approx 2 \pi \frac{\left(Z_{+} e \Gamma a\right)^{2}}{\varepsilon^{2} \kappa^{3}}(1+\kappa \zeta), \quad \frac{a^{2}}{S} \int_{R}^{\infty}\left(\frac{\partial \Phi}{\partial r}\right)^{2} r^{2} d r \approx 2 \pi \frac{\left(Z_{+} e \Gamma a\right)^{2}}{\varepsilon^{2} \kappa}(1+\kappa \zeta)
$$

with $\zeta=\sum_{l=0}^{\infty} \zeta_{o l} e^{-\Omega_{l} t} S_{l}(\theta, \phi)+$ C.C. . Combining eqs (36A) and (37A) and recalling that $\Gamma_{\max }=a^{-2}$, we obtain an implicit relationship linking the surface tension $\sigma$ with the surface deformation $\zeta$ :

$\sigma \approx \sigma_{o}+\Gamma_{\max }\left[k_{B} T \log \left(1-\Gamma / \Gamma_{\max }\right)+\beta\left(\Gamma / \Gamma_{\max }\right)^{2}-\frac{\left(Z_{+} e \Gamma\right)^{2}}{2 \varepsilon \kappa \Gamma_{\max }}(1+\kappa \zeta)\right]$

The second and third term in the right hand side of eq (S38) do not explicitly depend on the potential $\Phi$ (they are formally identical to those found in the absence of electrostatic interactions, eq (S18)) with the $\Phi$-dependence of $\Gamma$ is accounted for by eq (S22). In turn, $\Phi$ near the interface is related to the surface deformation $\zeta$ through eq (S34). Developing eq.(S38) in power series of the surface deformation $\zeta$, yields up to the linear terms in $\zeta$ :

$\left.\sigma(\zeta) \approx \sigma\right|_{R_{o}}+\left[\left(\frac{\partial \sigma}{\partial \Gamma}\right)\left(\frac{\partial \Gamma}{\left.\partial \Phi\right|_{R}}\right)\left(\frac{\left.\partial \Phi\right|_{R}}{\partial \zeta}\right)+\left(\frac{\partial \sigma}{\partial \zeta}\right)\right]_{\zeta=0} \zeta$ 
where $\left.\sigma\right|_{R_{o}}$ is the surface tension of the un-deformed charged sphere:

$$
\left.\sigma_{\text {undeformed }} \equiv \sigma\right|_{R_{o}}=\sigma_{o}+\Gamma_{\max }\left[k_{B} T \log \left(1-\Gamma / \Gamma_{\max }\right)+\beta\left(\Gamma / \Gamma_{\max }\right)^{2}\right]-\frac{\left(Z_{+} e \Gamma\right)^{2}}{2 \varepsilon \kappa}
$$

By using the explicit expressions for $\sigma$ and $\Gamma$ (eqs (S38) and (S22), respectively), it can be easily seen that the second contribution in the right hand side of eq (S39) is much greater than the first one in the limit of large $R$, that is: $(\partial \sigma / \partial \zeta)_{\zeta=0} \gg\left[(\partial \sigma / \partial \Gamma)\left(\partial \Gamma /\left.\partial \Phi\right|_{R}\right)\left(\left.\partial \Phi\right|_{R} / \partial \zeta\right)\right]_{\zeta=0}$. Therefore, from eq (S39) we calculate the surface tension of a deformed charged spherical bubble:

$$
\left.\sigma(\zeta) \approx \sigma\right|_{R_{o}}-\left.\frac{\left(Z_{+} e \Gamma\right)^{2}}{2 \varepsilon} \zeta \quad \approx \sigma\right|_{R_{o}}-\frac{\left(Z_{+} e \Gamma\right)^{2}}{2 \varepsilon} \sum_{l=0}^{\infty} \zeta_{o l} S_{l}(\theta, \phi)
$$

Notice that: a) even for an un-deformed body, the surface tension $\left.\sigma\right|_{R_{o}}$ depends on surface charging (see eq (S40)), a well-known effect reported in many textbooks) and, more important: b) the surface tension is not constant over a deformed interface but it is curvature-dependent.

Inserting eq (S41) into eq (S10), we get a modified dispersion relationship: $\left[-P_{\text {in }}-p_{\text {in }}+2 \mu_{\text {in }} \frac{\partial u_{r}^{\text {in }}}{\partial r}+P_{\text {out }}+p_{\text {out }}-\mu_{\text {out }} \frac{\partial u_{r}^{\text {out }}}{\partial r}\right]_{r=R_{o}}=\sigma(\zeta) H, \quad$ where: $\quad H=-\frac{2}{R_{o}}+\frac{1}{R_{o}^{2}}\left(2+\nabla_{\perp}^{2}\right) \zeta+$ $O\left(R_{o}^{-3}\right)$ (see eq (S11)). Retaining only linear terms in $\zeta$, eventually we obtain after simple algebra:

$$
\begin{aligned}
& P_{\text {in }}-P_{\text {out }}=\frac{2 \sigma_{\text {undeformed }}}{R_{o}} \quad \text { ( S42a ) } \\
& \left(\rho_{\text {in }}(l+1)+\rho_{\text {out }}\right) \Omega_{l}^{2}-\frac{2}{R_{o}^{2}}\left(\mu_{\text {in }}(l+1) l(l-1)+\mu_{\text {out }}(l+2)(l+1) l\right) \Omega_{l}+\frac{\sigma_{\text {app }}}{R_{o}^{3}}(l+2)(l+1) l(l-1)=0
\end{aligned}
$$

Equations (S42) are similar to eqs (S12), the only difference lying in the different surface tensions. Specifically, in the Young-Laplace equation for charged systems (eq (S42a)), $\sigma$ is replaced by $\sigma_{\text {undeformed }}=\left.\sigma\right|_{R_{o}}$ (the surface tension of an un-deformed charged bubble). Moreover, in the dispersion relationship for charged systems, eq (S42b), $\sigma$ is replaced by an "apparent" surface tension, $\sigma_{a p p}$, given by: 


$$
\sigma_{\text {app }}=\left(\sigma_{\text {undeformed }}-\frac{\left(Z_{+} e \Gamma\right)^{2}}{(l+2)(l-1) \varepsilon} R_{o}\right)
$$

Solution to eq.(S42b) with respect to $\Omega_{l}$ yields the bubble eigen-frequencies in the presence of charged surfactants adsorbed on its surface. Without loss of generality, we may write to the lowest order:

$\omega_{o l}=\operatorname{Im} \Omega_{l}=\left[\frac{1}{\rho_{\text {out }} R_{o}^{3}}(l+2)(l+1)(l-1)\left(\sigma_{\text {undeformed }}-\frac{\left(Z_{+} e \Gamma\right)^{2}}{(l+2)(l-1) \varepsilon} R_{o}\right)\right]^{1 / 2} \quad$ for $\quad l \geq 2$

If we consider an anchored bubble, the right hand side of eq (S44) must be multiplied by the corrective term, $D(l, a)$, defined after eqs (1) of the main text. Equation (S44) is coincident with eq (8a) of the main text where the shorthand notations $\rho=\rho_{\text {out }}$ and $\sigma=\sigma_{\text {undeformed }}$ have been used. Again, we want to stress that eq (S44) applies in the limit of small electrostatic potentials.

\section{D) SURFACTANTS SORPTION KINETICS IN THE PRESENCE OF STRONG INTERACTIONS AMONG THE ADSORBATES.}

Let us describe in detail the relationship between the fraction of adsorbed molecules $\Gamma^{\prime} / \Gamma_{n a x}$ and $C \equiv C(\tau)$ defined as the dimensionless bulk diffusant concentration calculated within a tiny layer near the interface (the so-called subphase). In the presence of both saturation and cooperativity effects among the adsorbates, a popular relationship is provided by the Frumkin adsorption isotherm. If we consider also the effect of the electrostatic field stemming from the charged surfactants, the Davies isotherm must be replaced by the Frumkin one. In the limit of weak surface potentials, however, we obtain a formula formally identical to that of Frumkin, but with a rescaled $\beta$-coefficient, $\beta_{\text {eff }}$ say. Under this approximation, eq (5) of the main text is rewritten as:

$$
\frac{\Gamma / \Gamma_{\max }}{1-\Gamma / \Gamma_{\max }} \exp \left(-\frac{2 \beta_{\text {eff }}}{k_{B} T} \frac{\Gamma}{\Gamma_{\max }}\right)=K C
$$

Notice that in eq.(S45) we replaced $\bar{C}$ (the surfactant concentration at infinite distance from the surface) by $C \equiv C(\tau)$ (the surfactant concentration in the subphase), a customarily assumption in adsorption kinetics models. The parameter $\beta_{\text {eff }}$ accounts for the intermolecular interactions among the molecules (proteins) 
adsorbed at the bubble surface. This term is responsible for the phase transition behavior of the adsorbate from a gas-like to a liquid-like configuration (the so-called GL transition) as shown in Figure S2 in the case of strong cooperative effects ( $\left.2 \beta_{e f f} / k_{B} T \equiv \alpha>>1\right)$.

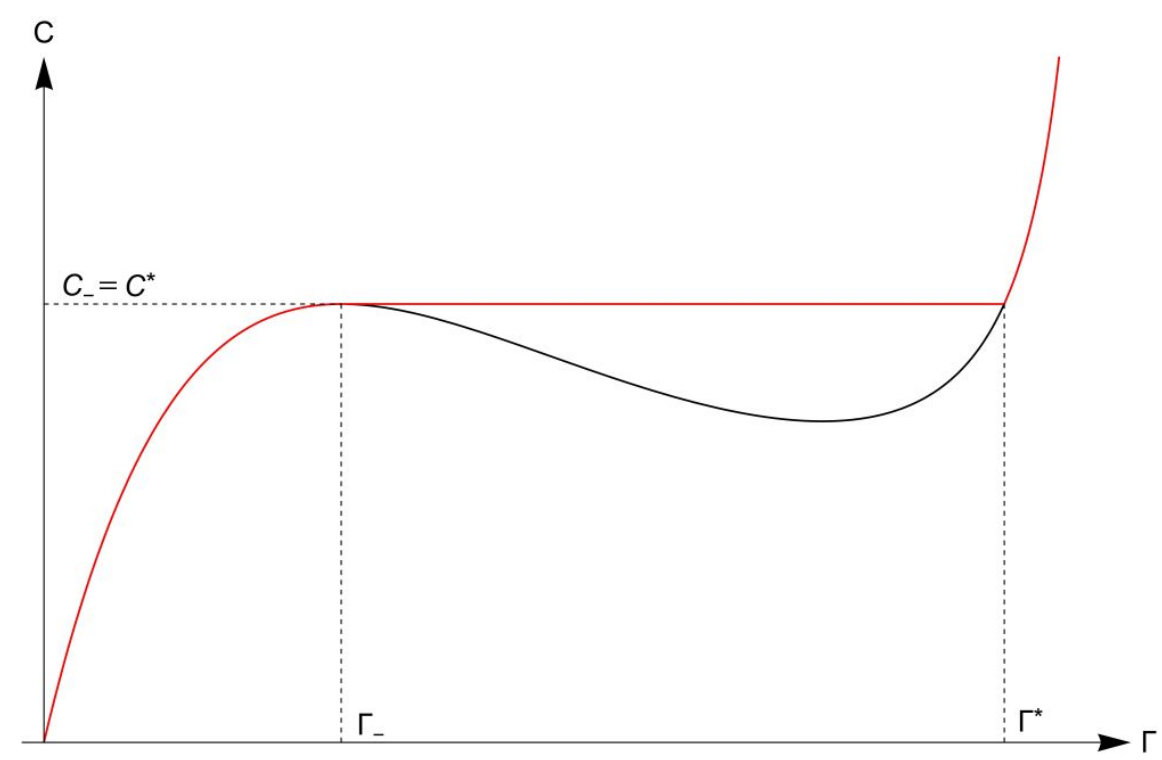

Figure S2. Concentration $\mathrm{C}$ vs surface excess $\Gamma$ in the case of strong cooperativity $(\alpha>>1)$ among the adsorbates. Black curve: mathematical solution, Red Curve: physical behavior. Below $\Gamma_{-}$and after $\Gamma^{*}$ black and red curves are coincident.

Since $C$ and $\Gamma^{\prime}$ must be single-valued functions, the unphysical concavity observed in Figure S2 must be replaced by a straight line (the red one). Physically, this describes first-order jump of $\Gamma^{\prime}$ from a rather low value, $\Gamma^{\prime}$ - say, to a very large surface coverage, $\Gamma^{*}$ say, for a tiny increase of $C$. Simple algebra shows that the maximum of the function (S45) occurs at: $\Gamma_{-}^{\prime}=\frac{1}{2}\left[1-\sqrt{1-\frac{4}{\alpha}}\right]$ and the corresponding value of $C_{,} C_{-}$say, is: $C_{-}=\frac{1}{K} \frac{1-\sqrt{1-\frac{4}{\alpha}}}{1+\sqrt{1-\frac{4}{\alpha}}} \exp \left(-\frac{\alpha}{2}\left[1-\sqrt{1-\frac{4}{\alpha}}\right]\right)$. Visual inspection of Figure S2 shows that: $C_{-}=C^{*}$.

It is worth noting that for small cooperativity parameter $\alpha$ the curve of Figure S2 does not show neither maxima nor minima. 
To proceed further, we replace the Frumkin isotherm (S45) into the kinetic equation (9) of the main text and partition the time interval into three regions:

Domain I: $\quad 0<C<C_{-} \quad$ gas-like behavior of the adsorbate

Domain II: $\quad C_{-}<C<C^{*}(\alpha)$ transition region (coexistence of gas-like and liquid-like patches)

Domain III: $\quad C>C^{*}(\alpha) \quad$ liquid-like behavior of the adsorbate

Let us investigate separately the behavior of the adsorption kinetics for selfinteracting adsorbates (large $\alpha$ ) at small $t$ (gas-like behavior) and large $t$ (liquid-like behavior). We do not attempt to investigate the adsorption kinetics into the transition region because the large deviation from equilibrium conditions prevents one to adopt the quasi-steady state approximation adopted in all Ward-Tordai type models.

\section{Small t behavior}

When $t$ is small, also $C$ and $\Gamma$ are small quantities and the only phase of the adsorbate is the gas-like one. So, eq (S45) reduces to: $\Gamma / \Gamma_{\max } \approx K C$, which is nothing but the Henry adsorption isotherm already used in deriving eq (10) of the main text. Inserting this result into eq (6), expanding it in power series of $\Gamma$ and making use of eqs (1) and (9), we obtain to the leading order:

$\left.\omega_{0 l}^{2} \approx \omega_{o l}^{2}\right|_{\Gamma=0}-A_{1}(l) \sqrt{t}+A_{2}(l, \alpha) t+\ldots . . \quad t<t_{\text {iniz }}^{*}$

where $\left.\omega_{o l}^{2}\right|_{\Gamma=0}$ is the resonance frequency of the bare bubble, while $A_{1}(l)$ and $A_{2}(l, \alpha)$ are two positive constants defined by: $A_{1}(l) \equiv 2 \bar{C} \sqrt{\frac{D_{e f f}}{\pi}} k_{B} T D(l, a) \frac{(l-1)(l+1)(l+2)}{\rho R_{0}^{3}}$ and: $A_{2}(l, \alpha) \equiv \frac{k_{B} T}{2 \Gamma_{\max }^{2}}\left(\Gamma_{\max }+\alpha\right) D(l, a) \frac{(l-1)(l+1)(l+2)}{\rho R_{0}^{3}}$, with $\alpha \equiv 2 \beta_{\text {eff }} / k_{B} T \gg>1$. The applicability of eq (S46) lies in the range $0<t<t_{\text {iniz }}^{*}$, where $t_{\text {iniz }}^{*} \equiv t_{\text {iniz }}^{*}(\alpha)$ denotes the time required to induce the transition. Notice that in eq (S46) the coefficients of $\sqrt{t}$ and $t$ have opposite sign. This means the two terms partially compensate to each other and, in the case of strong cooperativity $\left(\alpha \equiv 2 \beta_{\text {eff }} / k_{B} T \gg>1\right)$ the variation of the resonance frequency with time could even increase with time. 


\section{Large $t$ behavior}

After the GL transition took place (at $t=t_{\text {fin }}^{*}$, say, $t_{\text {fin }}^{*}$ being the time required to complete the phase transition), the numerical value of $C$ does not appreciably varies with time, remaining near the figures calculated at the transition. Whence, for

$t$ near $t_{\text {fin }}^{*}$ we may set: $\left.C(\tau) \approx C(\tau)\right|_{\tau=t_{\text {fin }}^{*}}=C^{*}(\alpha)=\frac{1}{K} \frac{1-\sqrt{1-\frac{4}{\alpha}}}{1+\sqrt{1-\frac{4}{\alpha}}} \exp \left(-\frac{\alpha}{2}\left[1-\sqrt{1-\frac{4}{\alpha}}\right]\right)$ as calculated before. Such an assumption simplifies eq (9) of the main text that becomes after a simple rearrangement:

$$
-\Gamma_{\max } \log \left(1-\frac{\Gamma(t)}{\Gamma_{\max }}\right) \approx \sqrt{\frac{D_{e f f}}{\pi}}\left[2 \phi_{b} \sqrt{t}-C^{*} \int_{0}^{t} \frac{d \tau}{\sqrt{t-\tau}}\right]=2 \sqrt{\frac{D_{e f f}}{\pi}}\left[\phi_{b}-C^{*}\right] \sqrt{t}
$$

That is:

$$
\frac{\Gamma(t)}{\Gamma_{\max }}=1-e^{-\mu(\alpha) \sqrt{t}}
$$

where $\mu(\alpha) \equiv \frac{2}{\Gamma_{\max }} \sqrt{\frac{D_{e f f}}{\pi}}\left(\bar{C}-C^{*}(\alpha)\right)$. Making use of eqs. (6) and (9) of the main text, eventually we get:

$$
\sigma=\sigma_{o}-k_{B} T\left(\Gamma_{\max } \mu(\alpha) \sqrt{t}-\frac{1}{2} \alpha\left(1-e^{-\mu(\alpha) \sqrt{t}}\right)^{2}\right)
$$

Equation (S49) describes the variation of the interfacial tension $\sigma$ upon protein adsorption beyond the gas-liquid transition of the adsorbate.

For the sake of comparison with the short-time behavior, it is useful to write in eq (S49): $t=t_{f i n}^{*}+\left(t-t_{f i n}^{*}\right)$. Then, expanding it in power series of $\left(t-t_{f i n}^{*}\right) / t_{f i n}^{*}<<1$, we arrive at: $\sigma=\sigma_{t_{f \text { fin }}}-$ const $_{1}(\alpha) \cdot \frac{t-t_{f \text { fin }}^{*}}{t_{f \text { fin }}^{*}}$. Inserting this result into eqs $(1)$ of the main text, we find the searched relationship between the adsorption kinetics and the bubble oscillation frequencies:

$$
\left.\omega_{0 l}^{2} \approx \omega_{o l}^{2}\right|_{\Gamma=\Gamma^{*}}-B_{1}(l, \alpha) \frac{t-t_{f i n}^{*}}{t_{f i n}^{*}}+\ldots . \quad t>t_{f i n}^{*}
$$


with $B_{1}(l, \alpha) \equiv \frac{1}{2} k_{B} T \mu(\alpha) \sqrt{t_{f i n}^{*}}\left[\Gamma_{\max }-\alpha \cdot \exp \left(-\mu(\alpha) \sqrt{t_{\text {fin }}^{*}}\right)\left(1-\exp \left(-\mu(\alpha) \sqrt{t_{\text {fin }}^{*}}\right)\right)\right] D(l, a) \frac{(l-1)(l+1)(l+2)}{\rho R_{o}^{3}}$. and $\mu(\alpha) \equiv \frac{2}{\Gamma_{\max }} \sqrt{\frac{D_{e f f}}{\pi}}\left(\bar{C}-C^{*}(\alpha)\right)$.

\section{E) NUMERICAL CALCULATIONS}

In this section we report a few calculations on the "apparent" surface tension (see eq (S43) and eq (8a) of the main text: $\sigma_{\text {app }}=\left(\sigma_{\text {undeformed }}-\frac{\left(Z_{+} e \Gamma\right)^{2}}{(l+2)(l-1) \varepsilon} R_{o}\right)$. This formula is valid only for low coverage (small electrostatic potential).

We set: $\sigma_{\text {undeformed }} \approx \sigma_{o} \approx 70 \cdot 10^{-3} \mathrm{~N} / \mathrm{m}$ (see below). Moreover, $\left|Z_{ \pm}\right|=9$ for $\mathrm{BSA}$ at $\mathrm{pH}=$ 7.4, (Baler, K., Martin, O.A., Carignano, M.A., Ameer, G.A., Vila, J.A., Szleifer, I. Electrostatic unfolding and interaction of Albumin driven by $\mathrm{pH}$ changes: a molecular dynamics study. J. Phys. Chem. B 2014, 118, 921-930), e $\approx 10^{-19} \mathrm{C}$, $1 / \varepsilon=1 / 4 \pi \varepsilon_{o} \varepsilon \approx 10^{10} / 80 \quad \mathrm{~N} \cdot \mathrm{m}^{2} \cdot \mathrm{C}^{-2}, \quad R_{o}=5 \cdot 10^{-4} \quad \mathrm{~m}, \quad \Gamma_{\max }=a^{-2} \approx(1-2 \mathrm{~nm})^{-2}$ and $\quad l=2$ (quadrupolar mode). Using the above numerical estimates, the dimensionless coverage $\Gamma / \Gamma_{\max }$ required for the onset of a shape instability turns out to be as small as 0.01 . This is a reasonable estimate suggesting that, even for incomplete surface charging, the Rayleigh instability may easily occur.

The used approximation: $\sigma_{\text {undeformed }}=\sigma_{o}+\Gamma_{\max }\left[k_{B} T \log \left(1-\Gamma / \Gamma_{\max }\right)+\beta\left(\Gamma / \Gamma_{\max }\right)^{2}\right]-\frac{\left(Z_{+} e \Gamma\right)^{2}}{2 \varepsilon \kappa}$ $\approx \sigma_{o}$ has been numerically confirmed in the case $\Gamma / \Gamma_{\max }=10^{-2}$ by setting $T=300^{\circ} \mathrm{K}$ and the Debye length $1 / \kappa$ was that of pure water calculated by the formula: $1 / \kappa=0.3 / \sqrt{10^{-7}} \mathrm{~nm}$ (Israelachvili, J.N., Intermolecular and surface forces, Third edition Academic Press, San Diego, 2011). The approximation $\sigma_{\text {undeformed }} \approx \sigma_{o}$ holds even for strong protein-protein interactions $\left(\beta / k_{B} T=10\right)$.

\section{F) INTERACTION BETWEEN A MOBILE CHARGE DISTRIBUTION AND AN ELECTROSTATIC FIELD.}


The energy of interaction between a distribution of charges and an inhomogeneous electric potential follows the standard relationship:

$$
E_{\text {TOT }}=q \psi+\mu \nabla \psi+Q \nabla \nabla \psi+\ldots \ldots
$$

where $q, \mu$ and $Q$ are the net charge, the dipole and quadrupole moments generated by the charge distribution, $\psi$ is the applied potential and $\nabla$ is the gradient operator.

For the sake of simplicity, we consider a mono-dimensional dipolar oscillation mode of the bubble $(I=1)$ interacting with an uniform field (i.e., a homogeneous gradient of the external potential). In this special case only the second term in the rhs of eq (S51) contributes: ( $q \psi=0$ and: $Q \nabla \nabla \psi=0)$, whence, eq.(S51) reduces to: $E_{T O T}=\mu \nabla \psi=\mu_{z} E_{z}$, where $\mu_{z}$ is the dipole moment induced by the charged bubble oscillation along the z-axis and $E_{z}$ is the z-component of the electric field. If we further assume that the strength of the dipole may change in order to maximize the electrostatic interaction, we may write: $\mu_{z}=\mu_{z}^{o}+\alpha_{z z} E_{z}$, where $\mu_{z}^{o}$ is the bubble dipole moment without surface charge redistribution while $\alpha_{z z}$ measures the efficiency of charge redistribution under the effect of an electric field. $\alpha_{z z}$ is strongly related to the mobility of the bubble adsorbed charges and it is inversely related to the frequency $\omega$ of the oscillating field, tending to zero when $\omega \rightarrow \infty$. Combining the above equations, we find: $E_{T O T}=\mu_{z} E_{z}+\frac{1}{2} \alpha_{z z} E_{z}^{2}$ (the factor $1 / 2$ arises by considering the work done to polarize a charge distribution). Inserting this expression into the formula for the oscillation amplitudes (eq.(3) of the main text), eventually we obtain at

resonance:

$$
\left(<A_{l}^{2}>\right)^{1 / 2} \propto \frac{\text { const }}{\gamma_{l} \omega_{o l}}\left(\mu_{z}^{o} E_{z}+\frac{1}{2} \alpha_{z z}\left(\omega_{o l}\right) E_{z}^{2}\right)
$$

The experiments reported in Figure S3 investigate the oscillation amplitudes against the strength of the applied field (expressed as Volt/interelectrode distance). The data show an excellent linearity between amplitudes and field suggesting a negligible polarization of the surface proteins distribution at resonance (of order $10^{2}$ $\mathrm{Hz}$ ). 

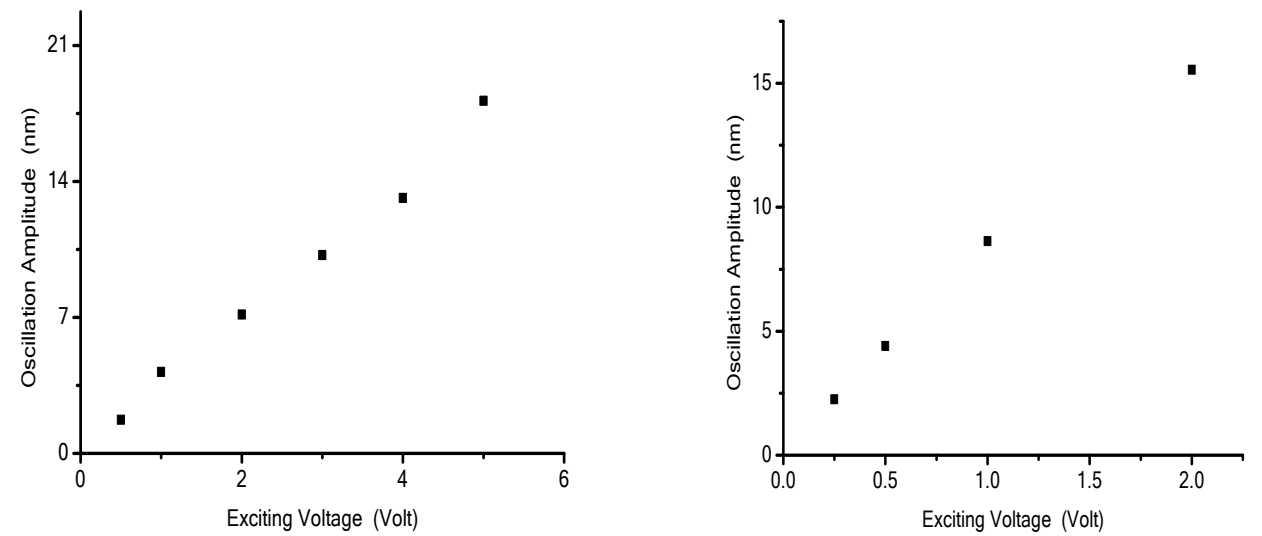

Figure S3. Left panel: oscillation amplitude versus exciting voltage at the electrodes measured at a BSA concentration below the critical concentration for the onset of the sphere to ellipsoid transition (see Figures 2). Right panel: as left panel but above the critical concentration. The interelectrode distance is $2 \mathrm{~mm}$. 